\title{
CONSIDERAÇÕES SOBRE A ENFERMAGEM NA ESCOLA E SUAS PRÁTICAS EDUCATIVAS
}

\author{
E. F. T. ROSA, E. C. OLIVEIRA* , I. C. M. CAMPOS, S. C. ANDRADE, I. C. ADÃO \\ Instituto Federal de Educação, Ciência e Tecnologia do Sudeste de Minas Gerais \\ ernani.coimbra@ifsudestemg.edu.br ${ }^{*}$
}

Submetido 04/11/2015 - Aceito 31/09/2017

DOI: $10.15628 /$ holos.2017.3644

\section{RESUMO}

A escola é um espaço favorável para educação em saúde, nela podem ser trabalhados diversos assuntos relativos à promoção de saúde, como: construção de hábitos saudáveis e identificação de agravos. A prática da enfermagem na saúde do escolar é contextualizada em uma política atual em desenvolvimento. Este trabalho tem o objetivo de Identificar através da pesquisa integrativa as contribuições para a compreensão da prática da enfermagem que atua na saúde do escolar e contribuir para o fortalecimento de uma política em plena implantação para atuação da enfermagem na saúde do escolar. Trata-se de uma revisão integrativa da literatura de caráter exploratório descritivo e comparativo, com abordagem qualitativa, cuja pesquisa foi realizada nas Bases de dados da Biblioteca Virtual de Saúde (BVS): LILACS, MEDLINE, SciELO e BDENF. Foram selecionados 18 artigos que indicam a participação do enfermeiro nas escolas. Os programas de saúde escolar são concepções novas, fundamentadas em um novo meio de promover saúde. O resultado do estudo permite refletir a participação do enfermeiro no contexto escolar, indica que o enfermeiro acrescenta em sua formação uma responsabilidade social, e deve utilizar de suas habilidades para promover saúde, independente do espaço físico.

PALAVRAS-CHAVE: Enfermagem, saúde escolar, educação em saúde

\section{CONSIDERATIONS ABOUT NURSING IN SCHOOL AND ITS PRACTICE EDUCATION}

\begin{abstract}
The school is a favorable space for health education, it can be worked various matters relating to the promotion of health, such as: building healthy habits and identification of diseases. The practice of nursing in the school health is contextualized in a current policy development. This work aims to identify through integrative research contributions to the understanding of nursing practice engaged in school health and contribute to the strengthening of a full deployment policy for nursing practice in health school. This is an integrative review of descriptive exploratory and comparative literature with a
\end{abstract}

qualitative approach, whose research was carried out in the Biblioteca Virtual de Saúde (BVS): LILACS, MEDLINE, SciELO and BDENF. They selected 18 articles that indicate the participation of nurses in schools. School health programs are new concepts, based on a new means of promoting health. The study results allows to reflect the participation of nurses in the school context, it indicates that nurses in their training adds a social responsibility, and should use their skills to promote health, regardless of the physical space.

KEYWORDS: Nursing, school health, health education 


\section{INTRODUÇÃO}

A escola, é o melhor local para praticar promoção em saúde, nela podem ser identificados agravos, trabalhar na prevenção de doenças e estimular comportamentos saudáveis desde a iniciação escolar. A maior parte dos problemas de saúde e até mesmo de comportamentos de riscos podem ser significativamente reduzidos, pois ao construir a escola como um espaço seguro e saudável, estaremos facilitando a prática de hábitos mais saudáveis.

Observamos que muitas inquietações nossas, já haviam se tornados grandes projetos, dentro e fora do Brasil, sendo que aqui no Brasil, o Governo Federal através do decreto no 6286 de 05 de dezembro de 2007, instituiu o Programa Saúde na Escola (PSE), voltado para escolas de educação básica. Este decreto mostra que apesar de existir uma preocupação inicial com o Governo Federal sobre ações de promoção, prevenção e atenção à saúde coloca como responsabilidade dos Secretários Municipais e Estaduais de Educação e Saúde a escolha dos municípios e escolas a serem atendidas pelo programa.

O enfermeiro neste contexto tem um papel social importante na promoção de saúde. Ele traz em sua formação, conteúdos curriculares, competências e habilidades que o tornam capaz de atuar em atividades de planejamento em saúde e tem o dever de conhecer, exigir e sugerir melhorias referentes à saúde.

\subsection{Promoção de saúde e escola}

Promoção de saúde é uma atividade concreta com a incumbência de pleitear ações que irão refletir em condições mais saudáveis de vida. Não existe um local específico para realizar promoção de saúde, o que se deve pensar é: como realizá-la? Quais os interesses e benefícios que esta prática irá trazer?

A carta de Ottawa, aprovada na Primeira Conferência Internacional sobre Promoção de Saúde em Ottawa em 21 de novembro de 1986, apresenta o seguinte conceito para promoção de saúde (Opas, 1986, sp):

\footnotetext{
A promoção da Saúde é o processo que visa aumentar a capacidade dos indivíduos e das comunidades para controlarem a sua saúde, no sentido de melhorá-la. Para atingir um estado de completo bem-estar físico, mental e social, o indivíduo ou o grupo devem estar aptos a identificar e realizar as suas aspirações, a satisfazer suas necessidades e a modificar ou adaptar-se ao meio.
}

Cabe ressaltar que esta é apenas uma definição, a promoção de saúde pode ser uma mudança do estilo de vida ou até mesmo um preparo para melhor qualidade de vida, seja em uma condição de doença, incapacidades ou até mesmo para as várias fases da vida. Isso ressalva, que se forem adquiridos hábitos saudáveis, desde a infância, poderiam ser mais fáceis manter estes hábitos na fase adulta.

Ainda abordando os reflexos que uma vida saudável pode trazer ao indivíduo e a comunidade, o Relatório do Encontro Internacional sobre Saúde em todas as Políticas, menciona os benefícios que uma boa saúde pode trazer, inclusive no crescimento econômico, pois uma pessoa mais saudável tem um melhor aprendizado e vai ter também melhor capacidade para trabalhar (Brasil, 2002). 
Um novo plano de resgatar os conceitos de promoção de saúde saíram das unidades básicas de saúde e foram levadas para dentro de escolas. Esse movimento é plenamente justificável devido ao fato de ser a escola um ambiente favorável, de aquisição de saber, criação de novos hábitos, diversidades sociais e culturais.

Os países europeus foram pioneiros neste tipo de política. Desde 1901, Portugal lidera os trabalhos de promoção de saúde escolar e hoje vem colhendo bons resultados, servindo de parâmetro para vários países. Em 2006, o Programa Nacional de Saúde Escolar em sua segunda edição, trouxe consigo várias evidências dos benefícios deste programa e de como ele funciona (Portugal, 2006).

Paralalelamente a isso, o processo de educação e saúde no âmbito escolar no Brasil vem realizando um trabalho mais tímido. Em 1996, por meio dos Parâmetros Curriculares Nacionais (PCN), foi incluído no currículo do ensino fundamental, a educação sexual. A visão de que o cuidado com o próprio corpo, a fim de criar hábitos mais saudáveis referentes à sexualidade, 0 tornou importante para inserção no currículo (Brasil, 1996). No entanto, a educação sexual era apenas um tema dentro de uma amplitude de temas que poderiam ser desenvolvidos referentes à temas de educação em saúde no contexto escolar, e ainda, não permeava ações de promoção de saúde por profissionais da área de saúde.

Em 2007, os programas de ação de saúde nas escolas ganharam força política com o decreto 6.286 que instituiu o Programa Saúde na Escola (PSE), relacionando o trabalho interministerial dos Ministérios da Saúde e Educação. Além de citar as ações de saúde que deveriam ser realizadas, colocou como competência das equipes de saúde da família e educação básica, a articulação destas ações (Brasil, 2007).

A partir desse momento, surgem então no Brasil, as Escolas Promotoras de Saúde, com a finalidade de identificar as prioridades de intervenção de saúde escolar e tornar o ambiente escolar um local para se praticar promoção em saúde. No entanto, vários paradigmas também surgiram e muitas escolas ainda não contam com ações de promoção em saúde em seu currículo. Isso faz com que muitas crianças e adolescentes não contem com estas práticas de acompanhamento de seu desenvolvimento e construção de hábitos saudáveis no âmbito escolar. (Brasil, 2007).

\subsection{Impasses encontrados}

Surge a partir do decreto que instituiu o Programa de Saúde na Escola (PSE), segundo o Instituto Brasileiro de Geografia e Estatística (IBGE) em 2008, a Pesquisa Nacional de Saúde do Escolar (PeNSE), com objetivo de monitorar as atividades e resultados dos trabalhos. Essa pesquisa identifica, por meio da Pesquisa Nacional por Amostra de Domicílio (PNAD), que 97\% das crianças de 15 e 19 anos frequentavam as escolas pesquisadas.

Estes dados refletem a importância de uma política de estratégia de promoção de saúde, levando a reafirmar a relevância do contexto saúde na escola como cita a Organização PanAmericana da Saúde (OPAS): "A escola é um espaço privilegiado por congregar, por um período importante, crianças e adolescentes numa etapa crítica de crescimento e desenvolvimento." (Opas, 2007 p.35) 
Através da Portaria 1861 de 04 de setembro de 2008, que instituiu os recursos financeiros para o PSE, observou-se que o referido programa não era aberto a todas as escolas públicas. As prioridades para sua adesão seriam as escolas que apresentassem $100 \%$ das equipes de saúde, ou seja, equipes completas e que apresentassem o Índice de Desenvolvimento da Educação Básica (IDEB) menor ou igual a 2,69 (Brasil, 2008).

Através do passo a passo para adesão ao PSE 2010, foi reafirmado que este, somente seria implantado nas condições supracitadas e que além de ser incorporado no Plano Político Pedagógico da escola, deveria ser enviado pelo município para a Elaboração do Projeto Municipal do PSE, para que o mesmo fosse enviado ao Ministério da Saúde (Brasil, 2010).

Infelizmente, a adesão a esse programa não ocorre de maneira homogênea, mesmo sendo um programa de atenção integral à saúde do escolar, e que deveria ocorrer dentro do princípio da equidade, o mesmo é executado por meio da adesão dos municípios, o se torna fator de limitação.

Em 2011, surgiu através da deputada Sueli Vidigal, o Projeto de Lei 1616/2011, que obrigava a contratação de no mínimo um profissional de enfermagem em creches e escolas, justificando que as estatísticas atribuem aos traumas físicos na faixa etária de zero a dez anos, uma das maiores causas de mortalidades nesta idade (Brasil, 2011).

Em resposta ao projeto, o Relator Deputado Geraldo Resende, justifica a não implantação da Lei pela falta de recursos para a contratação de profissionais, e relata: "É mais importante a presença desses profissionais de saúde em postos e centros de saúde." (Brasil, 2011).

Essa observação vai em contramão ao preconizado por especialistas que afirmam que a participação dos profissionais de saúde, inclusive a enfermagem, no cotidiano escolar, trazem benefícios a médio e longo prazo. Recomendam, inclusive a criação de parcerias com a comunidade e organizações de ensino para se contornar a falta de recursos financeiros. (Opas, 2007).

\subsection{O papel da enfermagem no contexto da saúde do escolar}

A falta de equidade exige o surgimento de ações inovadoras para detectar e sanar diferenças sociais, culturais e econômicas, a fim de melhorar o acesso à saúde. Esta identificação se faz necessária para permitir e garantir o acesso à informação e à saúde para todos.

Diversas atividades poderiam ser desenvolvidas pelo enfermeiro no âmbito escolar, desde o diagnóstico de situações de risco, propostas de correção e até mesmo atividades que promovam saúde na escola. O enfermeiro também poderia, segundo Rasche (2008), realizar no âmbito escolar: consultas de enfermagem, identificação de pré-disposição à agravos, cuidados curativos e preventivos. A parceria entre estes profissionais e os professores, possibilitaria até mesmo o preparo e aplicação de conteúdos que compõem algumas disciplinas.

À enfermagem não cabe somente o exercício da função assistencialista; ela deve assumir um papel ativo na gestão dos serviços de saúde e ainda, adquirir novas competências complementares para utilização de abordagem inovadora no processo de ensino-aprendizagem. 
Dessa forma, poderá envolver os sujeitos como atores no processo educativo da promoção de saúde.

Com vistas a essa temática, os objetivos desse trabalho foram: identificar através da pesquisa integrativa, as contribuições para a compreensão da prática escolar da enfermagem que atua na saúde do escolar e contribuir para o fortalecimento de uma política em plena implantação para atuação da enfermagem na saúde do escolar.

\section{METODOLOGIA}

Trata-se de um estudo de revisão integrativa da literatura de caráter exploratório, descritivo e comparativo com abordagem qualitativa.

Para a realização da pesquisa integrativa foi estabelecido a seguinte questão norteadora: "Dentre as pesquisas publicadas em periódicos nacionais, qual seria a participação do enfermeiro na saúde do escolar?"

Em seguida, foi realizada uma busca nas Bases de dados da Biblioteca Virtual de Saúde (BVS). Para o levantamento das publicações sobre a temática do estudo, utilizaram-se os seguintes descritores em português por meio dos operadores booleanos: enfermagem and saúde do escolar and educação em saúde.

A fim de definir a população iniciou-se a busca nos meses de setembro e outubro de 2015, nas bases de dados e portal virtual: LILACS (Literatura Latino Americana e do Caribe), MEDLINE (Medical Literatura Analysis), ScIELO (Scientific Electronic Library Online) e BDENF (Bases de Dados de Enfermagem), utilizando-se os seguintes critérios de elegibilidade: artigos na íntegra; idioma português; período compreendido entre 1995 a 2015.

A busca resultou em um total de 21 artigos, sendo que três foram excluídos por não relacionarem-se com o tema em discussão, resultando em uma amostra de 18 artigos.

A partir dos resultados encontrados, os artigos foram numerados e lidos com o auxílio de um instrumento de Coleta de Dados previamente elaborado pelos autores. Nesse momento, foram consideradas as variáveis: ano de publicação, título, base de dados, autores e suas formações, objetivos, região em que concerne a pesquisa e contribuições do estudo para esse trabalho.

\section{RESULTADOS E DISCUSSÃO}

Os artigos foram identificados por números para uma melhor abordagem e leitura dos quadros. Após identificação dos artigos em ordem numérica e análise das datas de publicações dos artigos, observou-se, conforme Quadro1 que foram apresentadas mais publicações no ano de 2007, somando três artigos. Referente aos anos em que estes artigos foram publicados, também foi observado, que a partir do ano de 2004, foram publicados 11 artigos, e no período que antecede 2004 foram somados 7 artigos.

Um marco internacional importante dessa avaliação diz respeito ao ano de 2004, início do 
Plano Nacional de Saúde (2004-2010) de Portugal, pioneiro em projetos governamentais de saúde do escolar desde 1901, repercutindo no Programa Nacional de Saúde Escolar (PNSE). Portugal não só se tornou referência para outros países, como também incluiu a educação para a saúde no currículo e possui atividade efetiva de saúde escolar.

No Brasil, devemos destacar o ano de 2007, com o surgimento do decreto 6286, de 05 de dezembro de 2007, que instituiu o Programa de Saúde Escolar (PSE), razão pela qual pode ter sido fonte de inspiração para novos estudos temáticos dessa questão.

Em relação às bases de dados, com busca comprometida até o dia 20 de outubro de 2015, foi observado que, MEDLINE e BDENF não tiveram artigos publicados referentes ao tema até o momento, e que no LILACS contou com apenas uma publicação, sendo que no ScIELO obtivemos 17 das amostras selecionadas.

Apesar de uma crescente discussão sobre a educação em saúde no contexto escolar, foram obtidas mais amostras na base de dados SCIELO, fato compreendido por se tratar de uma base de dados pública, o que nos leva a sugerir que as demais bases de dados diversifiquem mais seus temas publicados e insiram mais artigos relativos ao tema em discussão em seus arquivos.

Durante as pesquisas, foi observado que a formação acadêmica e a região onde eram realizadas as pesquisas, influenciavam significativamente no interesse pelo tema.

A fim de facilitar coleta de informações dos dezoito artigos selecionados foi necessário numerá-los, gerando o número de identificação de cada um.

Para descrição da formação dos autores, precisou-se fazer uma busca do Currículo Lattes dos mesmos, na Plataforma Lattes, devido à ausência de dados de formação profissional nos artigos, mesmo assim, dos 55 autores, 6 não foram identificados quanto à sua formação acadêmica. Das áreas de atuação dos 55 autores identificados, observou-se a participação de profissionais da educação (2), Ciências e saúde (1), Saúde pública (1), Psicólogo (2), fonoaudiólogo (2), médico (1), sociólogo (1), formação em desenho e plástica (1) sendo predominante a autoria de artigos por enfermeiros (39).

Um dos indicadores destes resultados se deve aos descritores utilizados na metodologia desta pesquisa: enfermagem and saúde do escolar and educação em saúde. Tendo um dos descritores específicos para enfermagem, pode ter direcionado artigos que descrevem somente o papel da enfermagem no contexto desta pesquisa, acabando por influenciar neste resultado.

Em relação ao grau de qualificação (titulação) dos profissionais descritos, observou-se que houve uma predominância por profissionais com doutorado, sendo que dos 55 autores, 27 correspondiam a doutores, 6 de pós-doutores, 6 de mestres, seguindo, de forma decrescente, os números de participação na autoria dos artigos por graduados de enfermagem, representando 5 autores, graduandos 04 autores e por fim, representando a classe com pós-graduações apenas 1 autor.

A participação de autores com titulação de pós-doutorados e doutores pode ser devido a afinidades a termos referentes à educação interligando-a no contexto saúde e as também numerosas pesquisas realizadas por esta classe. O graduado ou acadêmico de enfermagem representaram uma minoria de autores, o que nos leva inferir que o fato se deve à imaturidade 
profissional

As instituições do Sistema do Sistema de Educação Superior do País devem avaliar os projetos pedagógicos dos Cursos de Graduação de Enfermagem afim de que as competências e habilidades se façam presentes na formação destes profissionais. Para isso, instituiu-se a Resolução CNE/CES №3, de 7 de novembro de 2001, que no Art. 3 dispõe que o profissional qualificado Enfermeiro deverá ser:

Capaz de reconhecer e intervir sobre os problemas/situações de saúde-doença mais prevalentes no perfil epidemiológico nacional, com ênfase na sua região de atuação, identificando as dimensões biopsicossociais dos seus determinantes. Capacitado a atuar, com senso de responsabilidade social e compromisso com a cidadania, como promotor da saúde integral do ser humano (Brasil, 2001, p.1).

Neste sentido, faz se necessário refletir sobre o papel do enfermeiro como educador, independente de sua titulação e do papel que ele tem na promoção e proteção de saúde na vida do escolar. $O$ enfermeiro tem em sua formação subsídios que o torna qualificado para atuar na escola. Ele detém do conhecimento científico e também é um educador, o que lhe permite melhor abordagem dos temas de saúde.

Esperando justificar a falta de participação e até mesmo de conhecimento do enfermeiro sobre os projetos políticos de educação em saúde, fez se necessário pesquisar as regiões onde mais se preocupavam com a saúde do escolar.

Verifica-se ainda, uma predominância de pesquisas realizadas no estado de São Paulo, perfazendo 7 artigos, seguido pelo Rio Grande do Sul com três artigos, Rio de Janeiro com dois artigos e Rio Grande do Norte, Espírito Santo e Acre apresentando apenas um artigo cada um. Dois artigos entraram nesta avaliação por se tratarem de revisão bibliográfica sem definição territorial e o artigo com identificação no16 apesar de ter trabalhado com uma população definida, não mencionou a localidade durante a pesquisa.

Também pôde ser observado, que diversos estados não foram mencionados. Este pode ser um indicativo que além de influências na formação do Enfermeiro como dito anteriormente, também falta interesse da enfermagem e dos municípios na participação do PSE e adesão aos programas no binômio educação/saúde.

Por outro lado, pode ser que nestes outros estados, já exista um programa político atuante no processo de educação em saúde no contexto escolar, o que define outras prioridades de estudo. Porém, as experiências bem sucedidas poderiam incentivar a adesão de novos municípios, visto que o PSE apesar de existir um Decreto Federal de implantação, não prescinde da inscrição do município e, posteriormente, o Projeto Municipal, é um dos requisitos de adesão ao projeto, conforme a Portaria Interministerial no 3.696, de 25 de novembro de 2012 (Brasil, 2010).

Dos artigos selecionados tiveram alguns específicos, ou seja, que direcionavam a pesquisa a um único tema de saúde como: distúrbios na fala (1), orientação alimentar, (1), problemas visuais e auditivos (3), fracasso escolar (1), controle da Diabetes Mellitus (1). Apesar de um dos 
artigos retratar que a área da adolescência é pouco explorada pela adolescência foram achados (5) artigos, relacionados com uso de drogas e sexualidade.

Dos demais artigos (6), relacionaram a saúde de uma maneira geral no contexto escolar onde foram avaliados a formação do professor referentes a assuntos de saúde (2), experiências positivas de incentivo a outros profissionais da saúde e educação (3), opinião de escolares e educadores sobre saúde (1).

Os 18 artigos que visam apenas um achado de saúde no escolar, pode representar uma medida assistencialista e especializada, onde o escolar não é visto por completo. $O$ afunilamento dos temas que se colocam em pesquisas também pode ser responsável por este resultado.

Com relação à participação do enfermeiro na saúde do escolar foram identificadas várias práticas como: participação na capacitação de professores nos temas relacionados à saúde, promoção no autocuidado, prevenção de condutas de riscos, participação de projetos de educação em saúde, serviços de orientação sexual, contra o uso de drogas, educação alimentar, consultas de enfermagem, avaliação de deficiências auditivas, visuais, cognitivas, distúrbios de fala, controle de doenças crônicas, encaminhamento aos serviços de saúde e assistência adequada em situações emergentes.

\section{CONSIDERAÇÕES FINAIS}

Considerando que muitos enfermeiros desconhecem o programa de saúde escolar, este estudo buscou contribuir para a compreensão da enfermagem na escola com o objetivo ainda, de contribuir para o fortalecimento de uma política em implantação.

A prática da enfermagem na saúde do escolar é contextualizada em uma política atual em desenvolvimento, no Brasil, verifica-se que a promoção de saúde dentro das escolas está em processo de inicialização faltando ainda uma avaliação de impactos dentro do contexto: enfermagem na saúde do escolar.

Os corredores temáticos sugerem dimensões voltadas especificamente para a promoção de saúde. O desenvolvimento se dá muitas vezes com recuos e avanços e nada melhor do que seguir novos padrões gerenciais que comprovadamente demonstram mudanças até mesmo econômicas referentes ao custeio com a saúde.

Os resultados, não poderiam ser vistos imediatamente, mas com a consciência pública do direito à saúde e educação, participação dos gestores estaduais e municipais visando que o trabalho da enfermagem no contexto saúde do escolar, poderia beneficiar a promoção, proteção e até mesmo a recuperação da saúde.

Foi observado que a enfermagem precisa utilizar seus conhecimentos adquiridos para melhorar a qualidade da assistência, administrar e executar projetos políticos de saúde, e ainda compartilhar estas experiências, a fim de assumir a identidade da profissão e contribuir para implantação de novos projetos em benefício da saúde.

A regionalização dos acessos aos projetos de educação e saúde na escola nos levam a refletir mais sobre a participação dos outros estados que não aparecem nas pesquisas. Para o 
fortalecimento de uma política em plena implantação e que demonstra significativamente o papel do enfermeiro e suas práticas na área da educação é preciso que o enfermeiro participe na implantação destes trabalhos e divulguem mais as experiências vivenciadas no campo escolar.

Contudo, a enfermagem detém o saber em educação em saúde, ela pode no campo escolar identificar fatores predisponentes à agravos, trabalhar na adoção de condutas saudáveis pelos discentes e preparar um adulto mais saudável, com responsabilidade individual e coletiva para o bem comum.

\section{REFERÊNCIAS}

Brasil (2007). Decreto n.o 6.286, de 05 de dezembro de 2007. Institui o Programa de Saúde na Escola - PSE, e dá outras providencias. Diário Oficial [da] República Federativa do Brasil, Poder Executivo, Brasília, DF.

Brasil (1996). Ministério da Educação e do Desporto. Secretaria de Ensino Fundamental. Parâmetros Curriculares Nacionais. Brasília. 1996. Disponível em: <:http://www.mec.gov.br/sef/pcn.shtm>. Acesso em 15 outubro 2015.

Brasil (1999). Ministério da Educação. Parâmetros Curriculares Nacionais/ Ensino Fundamental: Orientação sexual. Brasília.

Brasil (2001). Ministério da Educação. Secretaria de Educação Superior. Diretrizes Curriculares para os Cursos de Graduação. Resolução CNE/ CES № 3, de 7 de novembro de 2001. [online]. Disponível em: <http://www.mec.gov.br/sesu/diretriz.htm> Acesso em 15 outubro 2015.

Brasil (2007). Ministério da Saúde. Escolas promotoras de saúde: experiências no Brasil. Brasília: Ministério da Saúde, v. 6.

Brasil (2002). Ministério da Saúde. As cartas da Promoção da Saúde. Brasília: MS, 2002. Recuperado em 15 de outubro de 2015, de http://www.saude.gov.br/con tratados.html

Brasil (2008). Ministério da Saúde. Portaria no 1.927/GM, de 17 de setembro de 2008, publicada no Diário Oficial da União no 181, de 18 de setembro de 2008, Seção 1, p. 59.

Brasil (2011). Ministério da Saúde. Portaria Interministerial no 3.696, de 25 de novembro de 2012, publicado no Diário Oficial da União no 152, de 08 de agosto de 2011, Seção 1, p. 49.

Brasil (2011). Ministério da Saúde. Portaria no 104, de 25 de janeiro de 2011. Dispões publicado no Diário Oficial da União no 18, de 26 de janeiro de 2011, Seção 1, p. 37.

Portugal (2006). Ministério da Saúde. Despacho n.o 12.045/2006, de 7 de Junho de 2006. Institui o Programa Nacional de Saúde do Escolar. Recuperado em 24 de agosto de 2015, de http://observatorio-lisboa.eapn.pt/ficheiro/Programa-Nacional-de-Sa\%C3\%BAde-Escolar2015.pdf

Organização Panamericana de Saúde (1986). Primeira Conferência Internacional sobre Promoção da Saúde-1986. Carta de Ottawa. Recuperado em 23 de agosto de 2015, de http://www.opas.org.br/promocao/upladArq/ottawa

Instituto Brasileiro de Geografia e Estatística. Pesquisa Nacional por Amostra de Domicílios 2008 (2008). Rio de Janeiro: IBGE, v. 29. Recuperado em 12 de agosto de 2015, de 
http://www.ibge.gov.br/home/estatistica/populacao/trabalhoerendimento/pnad2008/brasil pnad2008.pdf

Projeto de Lei n. 1616/11, de junho de 2011. Dispõe sobre a obrigatoriedade de manutenção de um profissional da área de enfermagem, enfermeiro ou técnico de enfermagem, nas unidades da rede pública de creches e escolas de educação infantil, e dá outras providências. Recuperado em 04 de setembro de 2015, de http://www.camara.gov.br/proposicoesWeb/prop_mostrarintegra?codteor $=889783$

Rasche, A. S. (2008). A atuação do enfermeiro escolar em projetos de promoção da saúde no Ensino Fundamental: 0 caso da 3 a Coordenadoria Regional de Educação no Rio Grande do Sul. (Dissertação de Mestrado). Universidade Federal do Rio de Janeiro, Rio de Janeiro, RJ, Brasil. 